\title{
SOS-BASED BLIND CHANNEL ESTIMATION UNDER SPACE-TIME BLOCK CODED TRANSMISSIONS
}

\section{Javier Vía *, Ignacio Santamaría}

\author{
Dept. of Communications Engineering \\ University of Cantabria \\ Santander, Cantabria 39005, Spain \\ e-mail: \{jvia,nacho\}@gtas.dicom.unican.es
}

\author{
Aydin Sezgin ${ }^{\dagger}$, Arogyaswami J. Paulraj \\ Information System Laboratory \\ Stanford University \\ Stanford, CA 94305 USA \\ e-mail: $\{$ sezgin,apaulraj\}@ stanford.edu
}

\begin{abstract}
In this paper, a new blind channel estimation technique for multipleinput multiple-output (MIMO) space-time block coded (STBC) systems is proposed. The technique is solely based on second-order statistics (SOS), and it consists on the extraction of the main eigenvector of a modified correlation matrix. Furthermore, it can be interpreted as a deterministic technique, i.e., in the absence of noise it is able to exactly recover the channel, up to a real scalar, within a finite number of observations. Unfortunately, in many practical cases there exist ambiguities associated to the problem of blind channel estimation from SOS. In order to resolve these problems we propose a new transmission technique, which is based on the combination of different STBCs (code diversity). In the simplest case, this technique reduces to a rotation or permutation of the transmit antennas (non-redundant precoding). Finally, the performance of the proposed method is demonstrated by means of some simulation examples.
\end{abstract}

\section{INTRODUCTION}

In the last ten years, space-time block coding (STBC) [1-4] has emerged as one of the most promising techniques to exploit spatial diversity in multiple-input multiple-output (MIMO) systems. This has motivated the development of different families of STBCs, including the orthogonal (OSTBCs) $[1,2]$, quasi-orthogonal (QSTBCs) $[3,4]$, and trace-orthogonal (TOSTBCs) $[5,6]$ space-time block codes. A common assumption for most of the STBCs is that perfect channel state information (CSI) is available at the receiver. Obviously, this is not true in practice, where the channel is usually estimated by means of training approaches, which implies a reduction in the bandwidth efficiency. On the other hand, the differential techniques $[7,8]$, which do not require channel knowledge at the receiver, incur a penalty in performance of at least $3-\mathrm{dB}$ as compared to the coherent maximum likelihood (ML) receiver. These shortcomings suggest the use of blind or semi-blind methods [9-12].

Blind techniques can be divided into two groups depending on whether they exploit the higher-order statistics (HOS) or the secondorder statistics (SOS) of the signals. The HOS-based methods include the optimal blind ML decoder, as well as several suboptimal methods [10], and in general, the computational complexity of HOSbased techniques remains relatively high. In order to overcome this

* This work was partially supported by MEC (Ministerio de Educación y Ciencia, Spain) under grant TEC2004-06451-C05-02 and FPU grant AP2004-5127.

${ }^{\dagger}$ The work of A.Sezgin is supported in part by the Deutsche Forschungsgemeinschaft (DFG) and by NSF Contract NSF DMS-0354674 ONR Contract ONR N00014-02-1-0088-P00006. problem several SOS-based methods have been proposed $[11,12]$. Specifically, in [11] the authors have proposed a subspace method for OSTBCs [2], and in [12] a more general blind decoding technique has been proposed.

In this paper we consider a general class of STBCs and propose a new blind channel estimation algorithm. The proposed technique is only based on SOS, and it can be seen as a deterministic approach, i.e., in the absence of noise it is able to recover the channel, up to a real scalar, within a finite number of observations. Furthermore, the proposed method generalizes the results in [11] and, unlike the technique in [12], it is not affected by more ambiguities than those associated to the problem of blind channel estimation from SOS. In order to avoid these remaining indeterminacy problems we propose a new transmission technique based on the idea of code diversity, which consists on the use of different STBCs in different blocks of data. Interestingly, this technique can be reduced to a non-redundant precoding consisting on a single rotation or permutation of the transmit antennas, which comes at virtually no computational expense at the transmitter. Finally, the performance of the proposed technique is evaluated by means of some numerical examples.

\section{DATA MODEL FOR SPACE-TIME BLOCK CODES}

In this paper, we will use bold-faced upper case letters to denote matrices, e.g., $\mathbf{X}$, with elements $x_{i, j}$; bold-faced lower case letters for column vector, e.g., $\mathbf{x}$, and light-faced lower case letters for scalar quantities. The superscripts $(\cdot)^{T}$ and $(\cdot)^{H}$ will denote transpose and Hermitian, respectively. The real and imaginary parts will be denoted as $\Re(\cdot)$ and $\Im(\cdot)$, and superscript $(\hat{\cdot})$ will denote estimated matrices, vectors or scalars. The trace, range (or column space) and Frobenius norm of matrix $\mathbf{A}$ will be denoted as $\operatorname{Tr}(\mathbf{A}), \operatorname{range}(\mathbf{A})$ and $\|\mathbf{A}\|$, respectively. Finally, the identity and zero matrices of the required dimensions will be denoted as $\mathbf{I}$ and $\mathbf{0}$, and $E_{n}[\cdot]$ will denote the expectation operator with respect to $n$.

A flat fading multiple-input multiple-output (MIMO) system with $n_{T}$ transmit and $n_{R}$ receive antennas is assumed. The $n_{T} \times n_{R}$ complex channel matrix is $\mathbf{H}=\left[\mathbf{h}_{1} \cdots \mathbf{h}_{n_{R}}\right]$, where $h_{i, j}$ denotes the channel response between the $i$-th transmit and the $j$-th receive antennas, and $\mathbf{h}_{j}=\left[h_{i, j}, \ldots, h_{n_{T}, j}\right]^{T}$. The complex noise at the receive antennas is considered both spatially and temporally white with variance $\sigma^{2}$.

Let us consider a linear space-time block code (STBC) transmitting $M$ symbols during $L$ time slots and using $n_{T}$ antennas at the transmitter side. The transmission rate is defined as $R=M / L$, and the number of real symbols transmitted in each block is $M^{\prime}=2 M$ 
for general complex codes and $M^{\prime}=M$ for real codes.

For a STBC, the $n$-th block of data can be expressed as

$$
\mathcal{S}(\mathbf{s}[n])=\sum_{k=1}^{M^{\prime}} \mathbf{C}_{k} s_{k}[n]
$$

where $\mathbf{s}[n]=\left[s_{1}[n], \ldots, s_{M^{\prime}}[n]\right]^{T}$ contains the $M^{\prime}$ real information symbols transmitted in the $n$-th block, and $\mathbf{C}_{k} \in \mathbb{C}^{L \times n_{T}}$, $k=1, \ldots, M^{\prime}$, are the STBC code matrices. In the case of real STBCs, the transmitted matrix $\mathcal{S}(\mathbf{s}[n])$ and the code matrices $\mathbf{C}_{k}$ are real.

The combined effect of the STBC and the $j$-th channel can be represented by the $L \times 1$ complex vectors

$$
\mathbf{w}_{k}\left(\mathbf{h}_{j}\right)=\mathbf{C}_{k} \mathbf{h}_{j}, \quad k=1, \ldots, M^{\prime},
$$

and defining $\tilde{\mathbf{w}}_{k}\left(\mathbf{h}_{j}\right)=\left[\Re\left(\mathbf{w}_{k}\left(\mathbf{h}_{j}\right)\right)^{T}, \Im\left(\mathbf{w}_{k}\left(\mathbf{h}_{j}\right)\right)^{T}\right]^{T}$ we can write $\tilde{\mathbf{w}}_{k}\left(\mathbf{h}_{j}\right)=\tilde{\mathbf{C}}_{k} \tilde{\mathbf{h}}_{j}$, where $\tilde{\mathbf{h}}_{j}=\left[\Re\left(\mathbf{h}_{j}\right)^{T}, \Im\left(\mathbf{h}_{j}\right)^{T}\right]^{T}$, and

$$
\tilde{\mathbf{C}}_{k}=\left[\begin{array}{cc}
\Re\left(\mathbf{C}_{k}\right) & -\Im\left(\mathbf{C}_{k}\right) \\
\Im\left(\mathbf{C}_{k}\right) & \Re\left(\mathbf{C}_{k}\right)
\end{array}\right]
$$

The signal at the $j$-th receive antenna is

$$
\mathbf{y}_{j}[n]=\mathcal{S}(\mathbf{s}[n]) \mathbf{h}_{j}+\mathbf{n}_{j}[n]=\sum_{k=1}^{M^{\prime}} \mathbf{w}_{k}\left(\mathbf{h}_{j}\right) s_{k}[n]+\mathbf{n}_{j}[n],
$$

where $\mathbf{n}_{j}[n]$ is the white complex noise with variance $\sigma^{2}$.

Defining now the vectors $\tilde{\mathbf{y}}_{j}[n]=\left[\Re\left(\mathbf{y}_{j}[n]\right)^{T}, \Im\left(\mathbf{y}_{j}[n]\right)^{T}\right]^{T}$ and $\tilde{\mathbf{n}}_{j}[n]=\left[\Re\left(\mathbf{n}_{j}[n]\right)^{T}, \Im\left(\mathbf{n}_{j}[n]\right)^{T}\right]^{T}$, the above equation can be rewritten as

$$
\tilde{\mathbf{y}}_{j}[n]=\sum_{k=1}^{M^{\prime}} \tilde{\mathbf{w}}_{k}\left(\mathbf{h}_{j}\right) s_{k}[n]+\tilde{\mathbf{n}}_{j}[n]=\tilde{\mathbf{W}}\left(\mathbf{h}_{j}\right) \mathbf{s}[n]+\tilde{\mathbf{n}}_{j}[n],
$$

where $\tilde{\mathbf{W}}\left(\mathbf{h}_{j}\right)=\left[\tilde{\mathbf{w}}_{1}\left(\mathbf{h}_{j}\right) \cdots \tilde{\mathbf{w}}_{M^{\prime}}\left(\mathbf{h}_{j}\right)\right]$. Finally, stacking all the received signals into $\tilde{\mathbf{y}}[n]=\left[\tilde{\mathbf{y}}_{1}^{T}[n], \ldots, \tilde{\mathbf{y}}_{n_{R}}^{T}[n]\right]^{T}$, we can write

$$
\tilde{\mathbf{y}}[n]=\tilde{\mathbf{W}}(\mathbf{H}) \mathbf{s}[n]+\tilde{\mathbf{n}}[n],
$$

where $\tilde{\mathbf{W}}(\mathbf{H})=\left[\tilde{\mathbf{W}}^{T}\left(\mathbf{h}_{1}\right) \cdots \tilde{\mathbf{W}}^{T}\left(\mathbf{h}_{n_{R}}\right)\right]^{T}$, and $\tilde{\mathbf{n}}[n]$ is defined analogously to $\tilde{\mathbf{y}}[n]$.

If $\mathbf{H}$ is known at the receiver, and assuming a Gaussian noise distribution, the information symbols can be optimally recovered by means of the maximum likelihood (ML) decoder, whose computational complexity depends on the specific STBC properties. In general, an alternative solution with a reduced computational complexity is given by the direct application of the linear minimum mean square error (LMMSE) criterion.

\section{PROPOSED BLIND CHANNEL ESTIMATION METHOD}

In this section we propose a new blind channel estimation technique based on the relaxed blind ML receiver. Although derived from a stochastic framework, the proposed method can be seen as a deterministic technique, i.e., in the absence of noise it is able to exactly recover, up to a real scalar, the MIMO channel. The method is based on a prewhitening of the observations and the extraction of the main eigenvector of a modified correlation matrix, and therefore, its computational complexity is relatively low. Let us start this section by introducing the main conditions of the proposed technique.

\subsection{Main Assumptions}

The proposed algorithm is based on the following mild assumptions:

Condition 1 (Number of available blocks) The MIMO channel is flat fading and constant during a period of $N \geq M^{\prime}$ transmission blocks.

Condition 2 (Input signals) The correlation matrix of the information symbols $\mathbf{R}_{\mathbf{s}}=E_{n}\left[\mathbf{s}[n] \mathbf{s}^{T}[n]\right]$ is full rank.

Condition 3 (Code properties) For some constant $c$, the code matrices satisfy $\sum_{k=1}^{M^{\prime}} \mathbf{C}_{k}^{H} \mathbf{C}_{k}=c^{2} \mathbf{I}$, which constitutes the necessary and sufficient condition for

$$
\|\tilde{\mathbf{W}}(\mathbf{H})\|=c\|\mathbf{H}\|,
$$

Condition 4 (Rate and number of receive antennas) The equivalent channel matrix $\tilde{\mathbf{W}}(\mathbf{H})$ is full column rank, which implies that the number of receive antennas satisfy

$$
n_{R} \geq\left\{\begin{array}{cl}
R & \text { for complex codes }, \\
R / 2 & \text { for real codes } .
\end{array}\right.
$$

Conditions 1 and 2 establish mild assumptions on the coherence time of the channel and the correlation properties of the inputs. The energy constraint in Condition 3 is directly related with the aim of reducing the effect of the channel fading, and therefore, it is satisfied by most of the practical STBCs. Finally, we must note that if $\tilde{\mathbf{W}}(\mathbf{H})$ is not full column rank, any information vector $\mathbf{s}[n]+\mathbf{z}[n]$, with $\mathbf{z}[n]$ belonging to the null subspace of $\tilde{\mathbf{W}}(\mathbf{H})$, will provide the same observations $\tilde{\mathbf{y}}[n]$ as $\mathbf{s}[n]$. Therefore, Condition 4 is a common assumption for most of the practical STBCs.

\subsection{Proposed Criterion}

Let us now introduce the blind maximum likelihood (ML) receiver, which is based on the minimization of the following cost function

$$
\mathcal{L}(\hat{\mathbf{H}}, \hat{\mathbf{s}}[n])=E_{n}\left[\|\tilde{\mathbf{y}}[n]-\tilde{\mathbf{W}}(\hat{\mathbf{H}}) \hat{\mathbf{s}}[n]\|^{2}\right],
$$

subject to the constraint that the estimated symbols $\hat{\mathbf{s}}[n]$ belong to some finite alphabet. Unfortunately, this is a fairly difficult problem, which is due to the fact that all the possible information symbol sequences have to be considered.

A direct simplification of the above problem is given by the relaxation of the finite alphabet constraint. Here, we must note that this relaxation introduces a real scalar ambiguity in the channel and signal estimates, which is a common ambiguity for all the SOSbased blind techniques. Therefore, from now on we will consider $\|\hat{\mathbf{H}}\|=\|\mathbf{H}\|=1$.

Considering the signal estimates $\hat{\mathbf{s}}[n]$ minimizing (1), the cost function can be rewritten as

$$
\mathcal{L}(\hat{\mathbf{H}})=E_{n}\left[\|\tilde{\mathbf{y}}[n]\|^{2}\right]-E_{n}\left[\tilde{\mathbf{y}}^{T}[n] \tilde{\mathbf{U}}(\hat{\mathbf{H}}) \tilde{\mathbf{U}}^{T}(\hat{\mathbf{H}}) \tilde{\mathbf{y}}[n]\right],
$$

where $\tilde{\mathbf{U}}(\hat{\mathbf{H}}) \in \mathbb{R}^{2 L n_{R} \times M^{\prime}}$ is an orthogonal basis for the subspace spanned by the columns of $\tilde{\mathbf{W}}(\hat{\mathbf{H}})$. Now, taking into account the property $\operatorname{Tr}\left(\mathbf{A}^{T} \mathbf{B}\right)=\operatorname{Tr}\left(\mathbf{A B}^{T}\right)$, with $\mathbf{A}, \mathbf{B} \in \mathbb{R}^{p \times q}$, the relaxed blind ML decoder is reduced to the following maximization problem

$$
\underset{\hat{\mathbf{H}}}{\operatorname{argmax}} \operatorname{Tr}\left(\tilde{\mathbf{U}}^{T}(\hat{\mathbf{H}}) \mathbf{R}_{\tilde{\mathbf{y}}} \tilde{\mathbf{U}}(\hat{\mathbf{H}})\right)
$$




\begin{tabular}{|c|c|c|c|c|c|c|c|c|c|}
\hline$\overline{n_{T}}$ & $P\left(n_{R}=1\right)$ & $P\left(n_{R}=2\right)$ & $P\left(n_{R}=3\right)$ & $P\left(n_{R}=4\right)$ & $P\left(n_{R}=5\right)$ & $\overline{P\left(n_{R}=6\right)}$ & $\overline{P\left(n_{R}=7\right)}$ & $\cdots$ & $P\left(n_{R}=15\right)$ \\
\hline 2 & $\overline{4}$ & & & & & & & & \\
\hline 3 & 6 & 4 & 2 & & & & & & \\
\hline 5 & 10 & 4 & 2 & & & & & & \\
\hline 6 & 12 & 8 & 4 & & & & & & \\
\hline 7 & 14 & 12 & 10 & 8 & 6 & 4 & 2 & & \\
\hline 8 & 16 & & & & & & & & \\
\hline 9 & 18 & 4 & 2 & & & & & & \\
\hline 10 & 20 & 8 & 4 & & & & & & \\
\hline 11 & 22 & 12 & 2 & & & & & & \\
\hline 12 & 24 & 16 & 8 & & & & & & \\
\hline 13 & 26 & 20 & 14 & 8 & 2 & & & & \\
\hline 14 & 28 & 24 & 20 & 16 & 12 & 8 & 4 & & \\
\hline 15 & 30 & 28 & 26 & 24 & 22 & 20 & 18 & $32-2 n_{R}$ & 2 \\
\hline 16 & 32 & & & & & & & & \\
\hline
\end{tabular}

Table 1. Identifiability characteristics for QSTBCs.

where $\mathbf{R}_{\tilde{\mathbf{y}}}=E_{n}\left[\tilde{\mathbf{y}}[n] \tilde{\mathbf{y}}^{T}[n]\right]=\tilde{\mathbf{W}}(\mathbf{H}) \mathbf{R}_{\mathbf{s}} \tilde{\mathbf{W}}^{T}(\mathbf{H})+\frac{\sigma^{2}}{2} \mathbf{I}$ is the correlation matrix of the observations.

Unfortunately, the dependency of $\tilde{\mathbf{U}}(\hat{\mathbf{H}})$ with $\hat{\mathbf{H}}$ is rather complicated, which precludes the direct solution of (2). However, we must note that the maximization problem in (2) is equivalent to

$$
\underset{\hat{\mathbf{H}}}{\operatorname{argmax}} \operatorname{Tr}\left(\tilde{\mathbf{U}}^{T}(\hat{\mathbf{H}}) \Phi_{\tilde{\mathbf{y}}} \tilde{\mathbf{U}}(\hat{\mathbf{H}})\right),
$$

where $\Phi_{\tilde{\mathbf{y}}}=\tilde{\mathbf{U}}(\mathbf{H}) \tilde{\mathbf{U}}^{T}(\mathbf{H})$ is the whitened and rank-reduced version (with rank $M^{\prime}$ ) of the correlation matrix $\mathbf{R}_{\tilde{\mathbf{y}}}$. Finally, taking Condition 3 into account, it can be proven in a straightforward manner that the above problems can be rewritten as

$$
\underset{\hat{\mathbf{H}}}{\operatorname{argmax}} \operatorname{Tr}\left(\tilde{\mathbf{W}}^{T}(\hat{\mathbf{H}}) \Phi_{\tilde{\mathbf{y}}} \tilde{\mathbf{W}}(\hat{\mathbf{H}})\right),
$$

which constitutes our final channel estimation criterion. Therefore, taking into account that $\Phi_{\tilde{\mathbf{y}}}=\tilde{\mathbf{U}}(\mathbf{H}) \tilde{\mathbf{U}}^{T}(\mathbf{H})$ is a projection matrix, we can state that the proposed criterion amounts to finding the MIMO channel $\hat{\mathbf{H}}$ maximizing the energy of the projection of $\tilde{\mathbf{W}}(\hat{\mathbf{H}})$ onto the subspace defined by the equivalent channel $\tilde{\mathbf{W}}(\mathbf{H})$.

\subsection{Algorithm Implementation and Further Discussion}

In practice, the theoretical correlation matrices $\mathbf{R}_{\tilde{y}}$ and $\boldsymbol{\Phi}_{\tilde{\mathbf{y}}}$ are not exactly known, and they have to be replaced by their finite sample estimates $\hat{\mathbf{R}}_{\tilde{y}}$ and $\hat{\mathbf{\Phi}}_{\tilde{\mathbf{y}}}$. Assuming a set of $N$ available blocks at the receiver, the finite sample estimate of $\mathbf{R}_{\tilde{\mathbf{y}}}$ is given by

$$
\hat{\mathbf{R}}_{\tilde{\mathbf{y}}}=\frac{1}{N} \sum_{n=0}^{N-1} \tilde{\mathbf{y}}[n] \tilde{\mathbf{y}}^{T}[n],
$$

and $\hat{\mathbf{\Phi}}_{\tilde{\mathbf{y}}}$ is obtained from the main $M^{\prime}$ eigenvectors of $\hat{\mathbf{R}}_{\tilde{\mathbf{y}}}$.

Let us now define the vectorized channel $\tilde{\mathbf{h}}=\left[\tilde{\mathbf{h}}_{1}^{T}, \ldots, \tilde{\mathbf{h}}_{n_{R}}^{T}\right]^{T}$, and the $M^{\prime}$ block diagonal matrices $\tilde{\mathbf{D}}_{k} \in \mathbb{R}^{2 L n_{R} \times 2 n_{T} n_{R}}$,

$$
\tilde{\mathbf{D}}_{k}=\left[\begin{array}{ccc}
\tilde{\mathbf{C}}_{k} & \cdots & \mathbf{0} \\
\vdots & \ddots & \vdots \\
\mathbf{0} & \cdots & \tilde{\mathbf{C}}_{k}
\end{array}\right], \quad k=1, \ldots, M^{\prime} .
$$

Thus, it is easy to see that the $k$-th column of $\tilde{\mathbf{W}}(\mathbf{H})$ is given by $\tilde{\mathbf{D}}_{k} \tilde{\mathbf{h}}$, and (3) can be rewritten as

$$
\underset{\hat{\mathbf{h}}}{\operatorname{argmax}} \hat{\tilde{\mathbf{h}}^{T} \hat{\boldsymbol{\Theta}}} \hat{\tilde{\mathbf{h}}}, \quad \text { with } \quad \hat{\boldsymbol{\Theta}}=\sum_{k=1}^{M^{\prime}} \tilde{\mathbf{D}}_{k}^{T} \hat{\mathbf{\Phi}}_{\tilde{\mathbf{y}}} \tilde{\mathbf{D}}_{k} .
$$

Finally, taking into account the energy constraint $\|\hat{\tilde{\mathbf{h}}}\|=1$, the channel estimate $\hat{\tilde{\mathbf{h}}}$ is directly given by the eigenvector associated to the largest eigenvalue of $\hat{\boldsymbol{\Theta}}$.

Here we must point out that, in the case of orthogonal spacetime block codes (OSTBCs), the proposed method is equivalent to the technique in [11], and the whitening step is not necessary, i.e., $\hat{\mathbf{\Phi}}_{\tilde{\mathbf{y}}}$ can be replaced by $\hat{\mathbf{R}}_{\tilde{\mathbf{y}}}$ [11]. This is due to the fact that, for OSTBCs, $\tilde{\mathbf{U}}(\hat{\mathbf{H}})=\tilde{\mathbf{W}}(\hat{\mathbf{H}}) /\|\hat{\mathbf{H}}\|$, which permits the direct solution of the maximization problem in (2). Moreover, the reformulation of the blind channel estimation problem as an eigenvalue problem makes the development of adaptive blind channel estimation algorithms very easy. Specifically, including a prewhitening of the observations $\tilde{\mathbf{y}}[n]$, the principal component analysis (PCA) algorithm in $[13,14]$ can be directly applied to obtain the solutions of (4).

\section{SOLUTION TO THE IDENTIFIABILITY PROBLEMS}

In this section, we analyze the identifiability conditions associated to the blind channel estimation process, and propose a new transmission technique to avoid the ambiguity problems. The proposed technique is based on the idea of code diversity, which consists on the use of different codes in different data blocks, but it can be reduced to a non-redundant precoding consisting on a set of rotations or permutations of the transmit antennas.

\subsection{Identifiability Problems}

Although some sufficient identifiability conditions have been obtained for the case of OSTBCs [15], the identifiability analysis for a wide class of STBCs is far from trivial. In this subsection we formulate the identifiability problem and derive some intuitive results, which will be later exploited to avoid some of the ambiguities.

The indeterminacies associated to the blind channel estimation from SOS are provoked by the existence of a MIMO channel $\hat{\mathbf{H}} \neq$ $c \mathbf{H}$ and signal $\hat{\mathbf{s}}[n] \neq c^{-1} \mathbf{s}[n]$, with $c$ a real scalar, satisfying

$$
\tilde{\mathbf{W}}(\hat{\mathbf{H}}) \hat{\mathbf{s}}[n]=\tilde{\mathbf{W}}(\mathbf{H}) \mathbf{s}[n], \quad n=0, \ldots, N-1 .
$$

It is easy to prove that, for a sufficiently large $N$, the above equality is equivalent to

$$
\operatorname{range}(\tilde{\mathbf{W}}(\hat{\mathbf{H}}))=\operatorname{range}(\tilde{\mathbf{W}}(\mathbf{H})) .
$$

Furthermore, we must point out that the solutions to (3) belong to the subspace defined by (5), and therefore, the ambiguities are associated to the blind channel estimation problem and not to the proposed 
criterion. Finally, from a practical point of view, the existence of spurious solutions is translated into a multiplicity $P>1$ of the largest eigenvalue of $\hat{\boldsymbol{\Theta}}$. Therefore, the $P$ principal eigenvectors of $\hat{\boldsymbol{\Theta}}$ constitute a basis $\mathbf{G} \in \mathbb{R}^{2 n_{T} n_{R} \times P}$ for the subspace containing all the solutions $\hat{\tilde{\mathbf{h}}}$ to the proposed blind channel estimation criterion.

Let us illustrate this with an example. Table 1 shows the multiplicities of the largest eigenvalue for the rate one QSTBCs introduced in $[3,4]$. As can be seen, the multiplicity decreases with the number of receive antennas $n_{R}$ until some fixed value, which is repeated (empty spaces in the table) for larger $n_{R}$. Unfortunately, these results suggest that the channel can not be unambiguously recovered from the SOS of the received signals.

\subsection{Code Diversity Technique}

From the identifiability discussion in the previous subsection we know that the true channel $\tilde{\mathbf{h}}$ belongs to the subspace defined by the matrix $\mathbf{G}(\mathbf{H}, \mathcal{C}) \in \mathbb{R}^{2 n_{T} n_{R} \times P(\mathcal{C})}$, where we have explicitly included the dependency on the channel $\mathbf{H}$ and the code $\mathcal{C}$.

Let us now consider $K$ different $\operatorname{codes} \mathcal{C}_{k}, k=1, \ldots, K$, with $n_{T}$ transmit antennas and transmitting $M\left(\mathcal{C}_{k}\right)$ information symbols in $L\left(\mathcal{C}_{k}\right)$ uses of the channel. Then, it is obvious that

$$
\tilde{\mathbf{h}} \in\left\{\operatorname{range}\left(\mathbf{G}\left(\mathbf{H}, \mathcal{C}_{1}\right)\right) \cap \cdots \cap \operatorname{range}\left(\mathbf{G}\left(\mathbf{H}, \mathcal{C}_{K}\right)\right)\right\},
$$

i.e., the true channel belongs to the intersection of the $K$ different subspaces, of size $P\left(\mathcal{C}_{k}\right)$, defined by the matrices $\mathbf{G}\left(\mathbf{H}, \mathcal{C}_{k}\right)$. However, in a general case, there is no reason to think that the rank of such intersection will be larger than 1, i.e., the spurious solutions to the blind channel estimation problem for code $\mathcal{C}_{k}$ do not necessarily maximize the criterion (3) when a different code $\mathcal{C}_{l}(l \neq k)$ is used.

The proposed technique is based on the previous idea. Assuming that the MIMO channel remains constant during a large enough interval, the first $M\left(\mathcal{C}_{1}\right)$ information symbols are transmitted during the first $L\left(\mathcal{C}_{1}\right)$ time slots using $\mathcal{C}_{1}$. In the following $L\left(\mathcal{C}_{2}\right)$ channel uses, $M\left(\mathcal{C}_{2}\right)$ new information symbols are transmitted by means of $\mathcal{C}_{2}$, and the same procedure is used with the $K$ STBCs. Thus, the set of $K$ STBC blocks can be considered as a composite STBC, and the proposed blind channel estimation technique can be directly applied. Furthermore, it is easy to prove [16] that the solutions $\hat{\tilde{\mathbf{h}}}$ to the channel estimation criterion belong to the intersection of the subspaces spanned by $\mathbf{G}\left(\mathbf{H}, \mathcal{C}_{k}\right)$, for $k=1, \ldots, K$.

\subsection{A Particular Solution: Non-Redundant Precoding}

Here we propose a particularly single code combination strategy, which is based on only one STBC which is modified by means of a non-redundant precoding consisting on the rotation of the transmit antennas. Specifically, considering $K$ different unitary matrices $\mathbf{Q}_{k}$ $(k=1, \ldots, K)$, and assuming a code $\mathcal{C}$ with transmission matrix $\mathcal{S}(\mathbf{s}[n], \mathcal{C})$, we define the following transmission matrices

$$
\mathcal{S}\left(\mathbf{s}[n], \mathcal{C}_{k}\right)=\mathcal{S}(\mathbf{s}[n], \mathcal{C}) \mathbf{Q}_{k}, \quad k=1, \ldots, K,
$$

which are associated to $K$ different codes $\mathcal{C}_{k}$. Thus, the code diversity is obtained by rotating the transmission matrix of one STBC and, since the effect of the rotations can be considered as part of the channel, the code properties are preserved. Finally, we must point out that the matrices $\mathbf{Q}_{k}$ can be chosen as permutation matrices, which does not increase the complexity of the transmitter and preserves the power properties associated to each transmit antenna.

\begin{tabular}{|c|c|c|}
\hline \hline$n_{T}$ & $P\left(n_{R}=1\right)$ & $P\left(n_{R}=2\right)$ \\
\hline \hline 2 & 4 & 4 \\
\hline 3 & 6 & 1 \\
4 & 8 & 1 \\
\hline 5 & 10 & 1 \\
6 & 12 & 1 \\
7 & 14 & 1 \\
8 & 16 & 1 \\
\hline 9 & 18 & 1 \\
10 & 20 & 1 \\
11 & 22 & 1 \\
12 & 24 & 1 \\
13 & 26 & 1 \\
14 & 28 & 1 \\
15 & 30 & 1 \\
16 & 32 & 1 \\
\hline \hline
\end{tabular}

Table 2. Application of the non-redundant precoding technique. QSTBC codes and $K=4$ random rotations.

\section{SIMULATION RESULTS}

In this section the performance of the proposed techniques is evaluated by means of some numerical examples. All the results are based on 5000 independent experiments. The MIMO channel is generated as a Rayleigh channel with unit variance elements, and the signal to noise ratio (SNR) is defined as the ratio between the averaged transmitted energy per symbol period and the noise variance. Finally, the i.i.d information symbols belong to a quadrature phase shift keying (QPSK) constellation, and the receivers have been designed based on the LMMSE criterion and a hard decision decoder.

In the first set of examples the non-redundant precoding technique, with $K=4$ precoding matrices, is evaluated. The precoding matrices have been randomly generated for each independent experiment. Table 2 shows the multiplicities ${ }^{1}$ of the largest eigenvalue of $\hat{\boldsymbol{\Theta}}$ for the rate one QSTBCs introduced in $[3,4]$. As can be seen, most of the ambiguities in Table 1 have been resolved, with the only exceptions of the MISO cases $\left(n_{R}=1\right)$ and the Alamouti code $\left(n_{T}=2\right)$ [1]. Interestingly, these results are analogous to those obtained in [11] for the case of OSTBCs.

In the second example, the QSTBC design for $n_{T}=M=L=$ 8 and $n_{R}=4$ has been evaluated. We have used $K=4$ permutation precoding matrices given by

$$
\begin{array}{ll}
\mathbf{Q}_{1}(8)=\left[\begin{array}{cc}
\mathbf{Q}_{1}(4) & \mathbf{0} \\
\mathbf{0} & \mathbf{Q}_{2}(4)
\end{array}\right], & \mathbf{Q}_{2}(8)=\left[\begin{array}{cc}
\mathbf{Q}_{3}(4) & \mathbf{0} \\
\mathbf{0} & \mathbf{Q}_{4}(4)
\end{array}\right], \\
\mathbf{Q}_{3}(8)=\left[\begin{array}{cc}
\mathbf{Q}_{1}(4) & \mathbf{0} \\
\mathbf{0} & \mathbf{Q}_{3}(4)
\end{array}\right], & \mathbf{Q}_{4}(8)=\left[\begin{array}{cc}
\mathbf{Q}_{2}(4) & \mathbf{0} \\
\mathbf{0} & \mathbf{Q}_{4}(4)
\end{array}\right],
\end{array}
$$

where

$$
\begin{array}{ll}
\mathbf{Q}_{1}(4)=\left[\begin{array}{llll}
1 & 0 & 0 & 0 \\
0 & 1 & 0 & 0 \\
0 & 0 & 1 & 0 \\
0 & 0 & 0 & 1
\end{array}\right], & \mathbf{Q}_{2}(4)=\left[\begin{array}{llll}
0 & 0 & 0 & 1 \\
1 & 0 & 0 & 0 \\
0 & 0 & 1 & 0 \\
0 & 1 & 0 & 0
\end{array}\right], \\
\mathbf{Q}_{3}(4)=\left[\begin{array}{llll}
0 & 0 & 0 & 1 \\
0 & 0 & 1 & 0 \\
0 & 1 & 0 & 0 \\
1 & 0 & 0 & 0
\end{array}\right], & \mathbf{Q}_{4}(4)=\left[\begin{array}{llll}
0 & 0 & 0 & 1 \\
1 & 0 & 0 & 0 \\
0 & 1 & 0 & 0 \\
0 & 0 & 1 & 0
\end{array}\right] .
\end{array}
$$

Figs. 1 and 2 show the MSE in the channel estimate and the bit error rate (BER) after decoding for different numbers $N$ of available blocks at the receiver. As can be seen, for a sufficiently large

\footnotetext{
${ }^{1}$ Excluding degenerated channels such as $\mathbf{H}=\mathbf{0}$, the multiplicities are independent of the specific channel realization.
} 


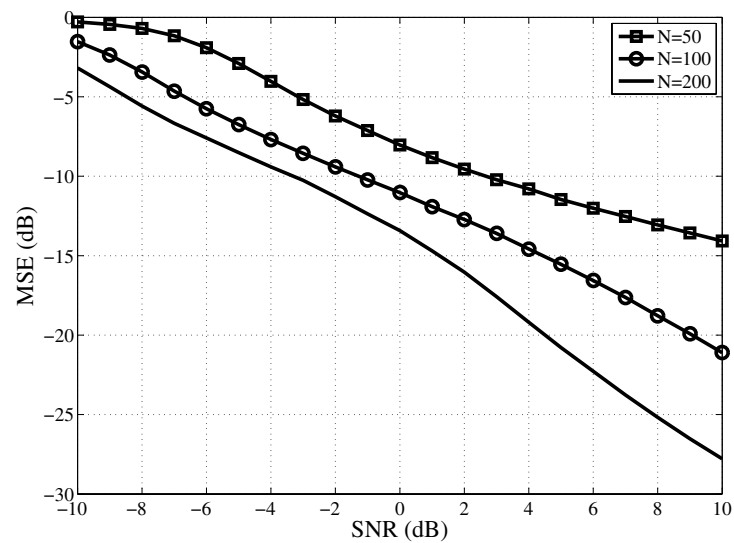

Fig. 1. Channel estimate MSE of the proposed method with $K=4$ code permutations. QSTBC with $n_{T}=M=L=8$ and $n_{R}=4$.

$N$, the performance degradation with respect to the coherent receiver is lower than the minimal loss (3-dB) associated to the QSTBC differential technique proposed in [8].

\section{CONCLUSIONS}

In this paper, a new blind channel estimation technique for multipleinput multiple-output (MIMO) space-time block coded (STBC) systems has been proposed. The technique is solely based on second order statistics (SOS), and therefore independent of the specific signal constellation. Furthermore, it does not require any assumption about the correlation matrix of the sources, which is translated in the fact that the technique can be seen as a deterministic approach, i.e., in the absence of noise it is able to exactly recover the channel, up to a real scalar, within a finite number of observations. Additionally, the channel identifiability conditions have been analyzed, and a general method to avoid the ambiguity problems has been proposed. The technique is based on the idea of code diversity, but it can be reduced to a non-redundant precoding consisting on the rotation or permutation of the transmit antennas. Finally, the performance of the proposed techniques has been illustrated by means of some numerical examples.

\section{REFERENCES}

[1] S.M. Alamouti, "A simple transmit diversity technique for wireless communications," IEEE J. Sel. Areas Comm., vol. 45, no. 9, pp. 1451-1458, 1998.

[2] V. Tarokh, H. Jafarkhani, and A. R. Calderbank, "Space-time block codes from orthogonal designs," IEEE Transactions on Information Theory, vol. 45, no. 5, pp. 1456-1467, 1999.

[3] H. Jafarkhani, Space-Time Coding: Theory and Practice, Cambridge University Press, 2005.

[4] A.Sezgin and T.J. Oechtering, "Complete characterization of the equivalent MIMO channel for quasi-orthogonal space-time codes," submitted to IEEE Trans. on Inf. Theory, 2004.

[5] J. K. Zhang, J. Liu, and K. M. Wong, "Trace-orthogonal full diversity cyclotomic space-time codes," in Space-Time processing for MIMO communications, A. B. Gershaman and N. D. Sidiropoulus, Eds. 2005, Wiley.

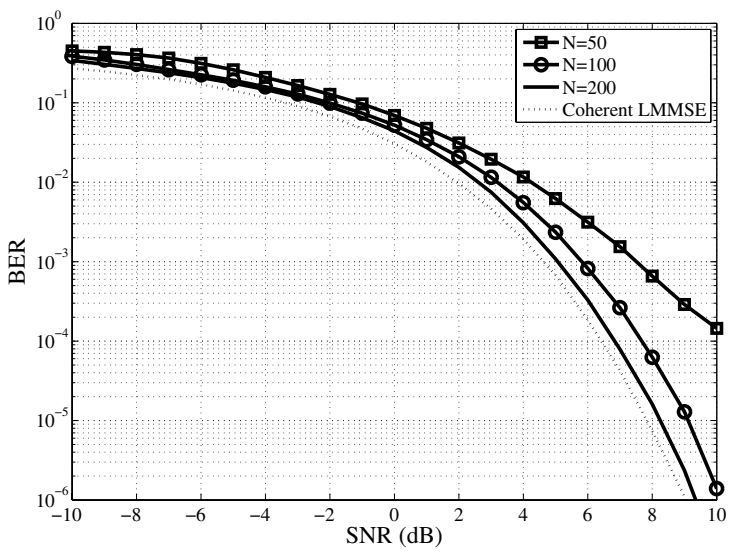

Fig. 2. Bit Error Rate (BER) after LMMSE decoding. QSTBC with $n_{T}=M=L=8$ and $n_{R}=4 . K=4$ code permutations.

[6] S. Barbarossa, Multiantenna Wireless Communication Systems, Artech House Publishers, 2005.

[7] G. Ganesan and P. Stoica, "Differential modulation using space-time block codes," IEEE Signal Processing Letters, vol. 9, no. 2, pp. 57-60, February 2002.

[8] Y. Zhu and H. Jafarkhani, "Differential modulation based on quasi-orthogonal codes," IEEE Transactions on Wireless Communications, vol. 4, no. 6, pp. 3005-3017, Nov. 2005.

[9] C. Budianu and L. Tong, "Channel estimation for space-time orthogonal block codes," IEEE Transactions on Signal Processing, vol. 50, no. 10, pp. 2515-2528, Oct. 2002.

[10] E.G. Larsson, P. Stoica, and J. Li, “Orthogonal space-time block codes: Maximum likelihood detection for unknown channels and unstructured interferences," IEEE Trans. on Signal Proc., vol. 51, no. 2, pp. 362-372, February 2003.

[11] S. Shahbazpanahi, A. B. Gershman, and J. H. Manton, "Closed-form blind MIMO channel estimation for orthogonal space-time block codes," IEEE Trans. Signal Processing, vol. 53, no. 12, pp. 4506-4517, Dec. 2005.

[12] L. Swindlehurst and G. Leus, "Blind and semi-blind equalization for generalized space-time block codes," IEEE Trans. on Signal Processing, vol. 50, no. 10, pp. 2489-2498, 2002.

[13] J. Vía, I. Santamaría, J. Pérez, and D. Ramírez, "Blind decoding of MISO-OSTBC systems based on principal component analysis," in IEEE International Conference on Acoustic, Speech, and Signal Processing, Toulouse, France, May 2006.

[14] J. Vía, I. Santamaría, and J. Pérez, "Blind identification of MIMO-OSTBC channels combining second and higher order statistics," in European Signal Processing Conference (EUSIPCO), Florence, Italy, September 2006.

[15] J. Vía and I. Santamaría, "Some results on the blind identifiability of orthogonal space-time block codes from second order statistics," in IEEE Int. Conf. on Acoustic, Speech, and Signal Proc. (ICASSP), Honolulu, Hawaii, USA, April 2007.

[16] J. Vía, A. Sezgin, A. J. Paulraj, and I. Santamaría, "Single and multiuser blind channel estimation under space-time block coded transmissions based on second order statistics," IEEE Trans. on Signal Proc. (Submitted), December 2006. 